\section{A long pain-free life} shown to be linked with a decrease in lifespan; however, the pathways and molecular mechanisms underlying this association are still unclear. and Caenorhabditis elegans now reveals a novel neuroendocrine pathway in which the activation of transient receptor potential cation channel subfamily $\mathrm{V}$ member 1 (TRPV1), which is pain-sensitive, regulates metabolism, with negative consequences for longevity.

The authors discovered that mice that lacked TRPV1 lived 100-130 days longer than their wild-type (WT) counterparts. Moreover, whereas WT mice showed a decline in their metabolic capacity with age, old $\operatorname{Tr} p v 1^{-/-}$mice retained a more youthful metabolism - they maintained a more efficient circadian transitioning between glucose and lipid metabolism, and a higher oxygen consumption rate. Glucose- and insulin-tolerance tests revealed that $\operatorname{Tr} p v 1^{-/-}$animals were not hyperinsulinaemic, but they did release more insulin following glucose challenge than did WT mice, possibly explaining their altered metabolism.

To elucidate how the mice that lacked TRPV1 maintained a youthful metabolic profile, the authors used various mutant strains of $C$. elegans. Worms with null mutations in two bers (TRPV mutants) lived approximately one-third longer than WT worms. Similarly, RNAi-mediated inactivation of $\operatorname{tax}-6$, the worm orthologue of the phosphatase calcineurin, also extended lifespan. However, inactivation of tax-6 in the (already long-lived) TRPV mutants did not
Chronic pain has previously been A new study by Riera et al. in mice genes encoding TRPV family mem- further extend longevity, leading the authors to conclude that TRPVs may function upstream of calcineurin.

Calcineurin is known to dephosphorylate CREB-regulated transcription co-activator 1 (CRTC1), resulting in the trafficking of CRTC1 from the cytosol into the nucleus. TRPV mutant worms that expressed a mutant form of CRTC-1 that was constitutively nuclear had a normal lifespan. Moreover, in WT mouse dorsal root ganglion (DRG) neurons, activation of TRPV1 with capsaicin (a natural TRPV1 agonist) led to shuttling of cytosolic CRTC1 to the nucleus; however, this capsaicininduced trafficking of CRTC1 was abrogated in $\operatorname{Tr} p v 1^{-/-}$neurons. Together, these data indicate that in WT neurons, TRPV1 activity promotes the localization of CRTC1 to the nucleus.

Trpv1 $1^{-1-}$ mouse DRG neurons had reduced $\mathrm{mRNA}$ levels for various CRTC1-induced genes, including the gene encoding calcitonin gene-related peptide (CGRP). Incubation of pancreatic $\beta$-cells with recombinant CGRP reduced the glucose-stimulated release of insulin. Strikingly, whereas in WT mice circulating levels of CGRP increased markedly with age, CGRP levels in Trpv1 $1^{-/-}$mice remained low even in older animals. Furthermore, unlike vehicle-treated aged mice, old WT mice that were treated with a CGRP antagonist for 6 weeks showed youthful metabolic profiles that were similar to those of old Trpv1 $1^{-/-}$mice. These results imply that activated TRPV1-positive DRG neurons release CGRP onto the pancreas and inhibit $\beta$-cell release of insulin.
In summary, this study provides evidence that pain-sensitive sensory neurons in the DRG release CGRP, which could over time have long-term consequences on metabolism and longevity by reducing pancreatic release of insulin. These findings may have important implications with regard to age-associated type 2 diabetes and chronic pain disorders.

Natasha Bray

This article originally appeared in Nature Rev. Neurosci. (http://dx.doi.org/10.1038/nrn3778).

ORIGINAL RESEARCH PAPER Riera, E. et al. TRPV1 pain receptors regulate longevity and metabolism by neuropeptide signaling. Cell 157 , 1023-1036 (2014)
CGRP levels in $\operatorname{Trpv} 1^{-1-}$ mice remained low even in older animals קפ
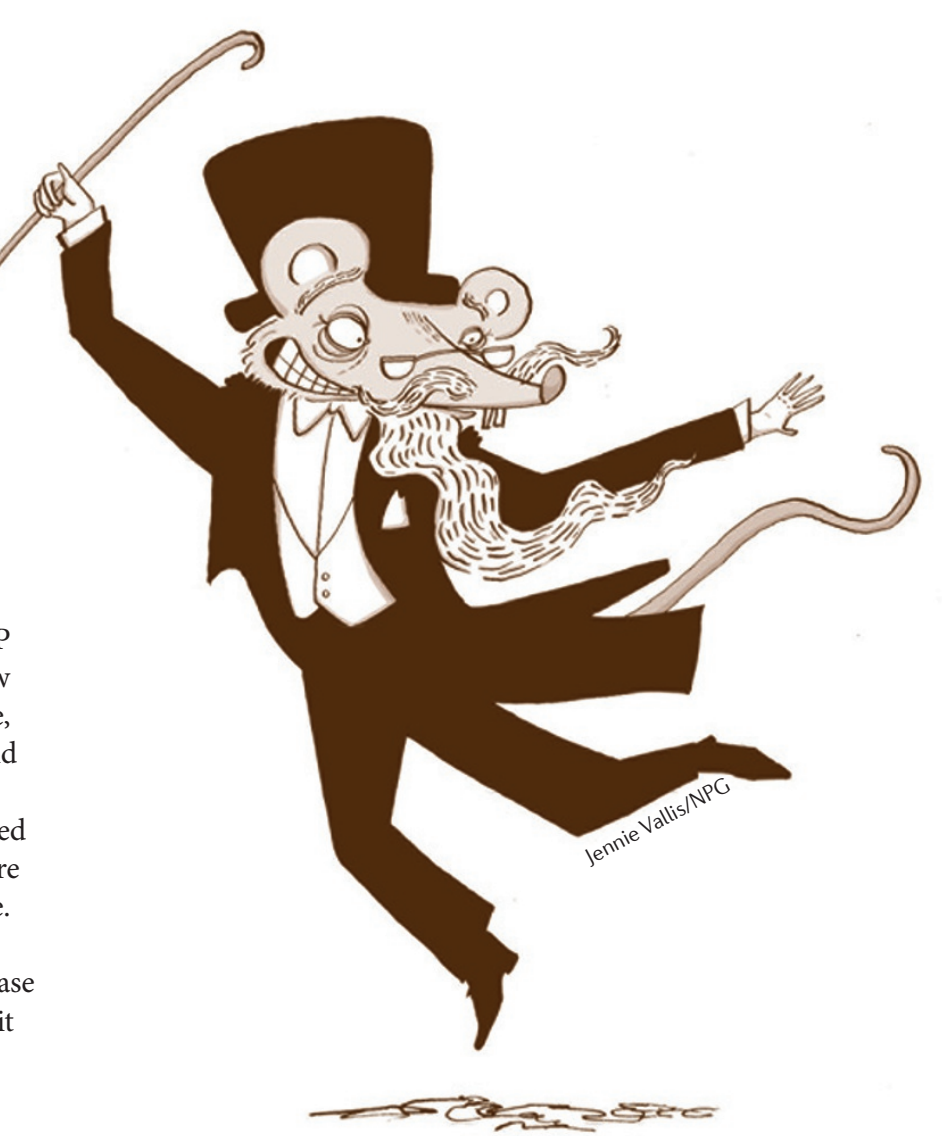